УДК 519.2

\title{
A Class of Local Linear Estimators with Functional Data
}

\author{
Sara Leulmi* \\ Fatiha Messaci ${ }^{\dagger}$ \\ Department of Mathematics \\ University brothers Mentouri \\ Road of Ain El Bey,Constantine, 25017
}

Algeria

Received 04.12.2018, received in revised form 28.01.2019, accepted 06.03.2019

We introduce a local linear nonparametric estimation for the generalized regression function of a scalar response variable given a random variable taking values in a semi metric space. We establish a rate of uniform consistency for the proposed estimators. Then, based on a real data set we illustrate the performance of a particular studied estimator with respect to other known estimators.

Keywords: locally modelled regression, Nonparametric estimation, Rate of Convergence, Uniform almost complete convergence.

DOI: 10.17516/1997-1397-2019-12-3-379-391.

\section{Introduction and preliminaries}

Since the pioneer works summarized in [6], several studies have dealt with the nonparametric functional estimation. This research field is motivated by the fact that several data collected in practice, are given in the form of curves. Moreover, the progress of the digital computing tools allows the treatment of such observations. Different kernel type estimators have been studied in the literature, see for example [6]. Then, inspired by the local linear nonparametric method, [1] have introduced a more general and flexible method than the kernel one. It is the so called local modelling approach. They obtain a rate of the pointwise almost-complete convergence for their estimator of the regression function.

But, as pointed out in [4] "the uniform consistency results are indispensable tools for the study of more sophisticated models in which multi-stage procedures are involved". Under uniform convergence, one can make prediction even if the data are not well observed. We also can solve some problems such as data-driven bandwidth choice (see [2]), or bootstrapping (see [5]). Uniform convergence of other local linear nonparametric estimators has been investigated in some papers as [3] and [8]. In this work, our principal aim is to establish the uniform almost complete convergence of the local linear estimator of a generalized regression function (which includes the estimator introduced in [1]) and to focus on a tool of prediction (a conditional quantile estimator). More precisely, Section 2 is devoted to introduce the generalized regression function estimator and to state its pointwise convergence. Section 3 contains the principal results of this work which consist to establish the rate of the uniform almost convergence of the last estimator and to focus on the particular case of the conditional distribution function estimation from which we deduce a rate of the uniform consistency of a conditional quantile estimator. In Section 4 using a real data set, the prediction obtained from this last estimator is compared to those of two other known estimators. Finally, the detailed proofs of some needed lemmas are evoked in the Appendix.

\footnotetext{
*math17sara@yahoo.fr

†f_messaci@yahoo.fr

(c) Siberian Federal University. All rights reserved
} 


\section{The estimation and the pointwise almost-complete convergence}

\subsection{The model}

Let us consider $n$ pairs of random variables $\left(X_{i}, Y_{i}\right)_{i=1, \ldots, n}$ independent and identically distributed as the pair $(X, Y)$ which is valued in $\mathcal{F} \times \mathbb{R}$, where $(\mathcal{F}, d)$ is a semi-metric space. Our goal is to estimate the generalized regression function, defined for all $x$ in $\mathcal{F}$, by

$$
m_{\varphi}(x)=E(\varphi(Y) \mid X=x),
$$

where $\varphi$ is a known real-valued borel function.

It is clear that $m_{\varphi}$ generalizes the classical regression function (set $\varphi(t)=t$ ) as well as the conditional distribution function (set for any $y \in \mathbb{R}, \varphi(t)=1_{]-\infty, y]}(t)$ ).

Following [1] who proved the pointwise almost complete convergence of the classical regression function estimator, the local linear estimate of $m_{\varphi}$ is obtained as the solution for $a$ of the following minimization problem

$$
\min _{(a, b) \in \mathbb{R}^{2}} \sum_{i=1}^{n}\left(\varphi\left(Y_{i}\right)-a-b \beta\left(X_{i}, x\right)\right)^{2} K\left(h^{-1} d\left(X_{i}, x\right)\right),
$$

where $\beta(.,$.$) is a known operator from \mathcal{F} \times \mathcal{F}$ into $\mathbb{R}$ such that, $\forall x \in \mathcal{F}, \beta(x, x)=0$, the function $K$ is a kernel and $h:=h_{n}$ is a sequence of strictly positive real numbers which plays a smoothing parameter role.

This approach assumes that $a+b \beta(., x)$ is a good approximation of $m_{\varphi}($.$) around x$. As $\beta(x, x)=0, a$ will be a suitable estimate for $m_{\varphi}(x)$.

By a simple calculus, one's can derive the following explicit estimator

$$
\widehat{m}_{\varphi}(x)=\frac{\sum_{i, j=1}^{n} W_{i j}(x) \varphi\left(Y_{j}\right)}{\sum_{i, j=1}^{n} W_{i j}(x)} \quad\left(\frac{0}{0}:=0\right),
$$

where

$$
W_{i j}(x)=\beta\left(X_{i}, x\right)\left(\beta\left(X_{i}, x\right)-\beta\left(X_{j}, x\right)\right) K\left(h^{-1} d\left(X_{i}, x\right)\right) K\left(h^{-1} d\left(X_{j}, x\right)\right) .
$$

As for $l \in\{0,1\}$, we have

$$
\begin{aligned}
& \sum_{i, j=1}^{n} W_{i j}(x) \varphi^{l}\left(Y_{j}\right)=\sum_{i<j}\left\{\left(\beta\left(X_{i}, x\right)-\beta\left(X_{j}, x\right)\right)\left(\beta\left(X_{i}, x\right) \varphi^{l}\left(Y_{j}\right)-\beta\left(X_{j}, x\right) \varphi^{l}\left(Y_{i}\right)\right) \times\right. \\
& \times K\left(h^{-1} d\left(X_{i}, x\right)\right) K\left(h^{-1} d\left(X_{j}, x\right)\right\},
\end{aligned}
$$

if the denominator of the estimator $\widehat{m}_{\varphi}(x)$ is zero, it is the same for its numerator. Moreover, under assumptions $(\mathrm{H} 1)$ and $(\mathrm{H} 3)-(\mathrm{H} 7)$, we get $E\left(W_{12}(x)\right)>0$ (see the proof of Lemma 4.4 in [1]).

Notice that the expression of $\widehat{m}_{\varphi}$ allows fast computational issue and that the choices of $\beta$ and $d$ will be crucial.

\subsection{The pointwise almost-complete convergence}

Let $x$ be a fixed point in $\mathcal{F}$, for any positive real $h, B(x, h):=\{y \in \mathcal{F} / d(x, y) \leqslant h\}$ denotes a closed ball in $\mathcal{F}$ of center $x$ and radius $h$. We also define $\Phi_{x}\left(r_{1}, r_{2}\right):=P\left(r_{1} \leqslant d(X, x) \leqslant r_{2}\right)$, where $r_{1}$ and $r_{2}$ are two real numbers.

We investigate the asymptotic behaviour of the local linear estimator $\widehat{m}_{\varphi}$, under the following assumptions. 
(H1) For any $h>0, \Phi_{x}(h):=\Phi_{x}(0, h)>0$.

(H2) $m_{\varphi} \in\left\{f: \mathcal{F} \rightarrow \mathbb{R}, \lim _{d\left(x, x^{\prime}\right) \rightarrow 0} f\left(x^{\prime}\right)=f(x)\right\}$.

(H2') $m_{\varphi} \in\left\{f: \mathcal{F} \rightarrow \mathbb{R}, \exists b>0, \forall x^{\prime} \in \mathcal{F} ;\left|f(x)-f\left(x^{\prime}\right)\right| \leqslant C_{x} d^{b}\left(x, x^{\prime}\right)\right\}$, where $C_{x}$ is a positive constant depending on $x$.

(H3) The function $\beta(.,$.$) is such that: \exists 0<M_{1}<M_{2}, \forall x^{\prime} \in \mathcal{F}$,

$$
M_{1} d\left(x, x^{\prime}\right) \leqslant\left|\beta\left(x, x^{\prime}\right)\right| \leqslant M_{2} d\left(x, x^{\prime}\right) .
$$

(H4) The kernel $K$ is a positive and differentiable function on its support $[0,1]$.

(H5) The bandwidth $h$ satisfies: $\lim _{n \rightarrow \infty} h=0$, and $\lim _{n \rightarrow \infty} \frac{\ln n}{n \Phi_{x}(h)}=0$.

(H6) There exists an integer $n_{0}$, such that

$$
\forall n>n_{0}, \forall x \in \mathcal{F}, \frac{1}{\left.\Phi_{x}(h)\right)} \int_{0}^{1} \Phi_{x}(z h, h) \frac{d}{d z}\left(z^{2} K(z)\right)>C>0
$$

and

$$
h \int_{B(x, h)} \beta(u, x) d P_{X}(u)=o\left(\int_{B(x, h)} \beta^{2}(u, x) d P_{X}(u)\right),
$$

where $d P_{X}$ is the distribution of $X$.

(H7) $\forall m \geqslant 2: x \rightarrow E\left(|\varphi(Y)|^{m} / X=x\right)$ is a continuous operator.

Remark that our hypotheses are very similar to the assumed conditions in [1].

Let us state the pointwise almost-complete convergence (a.co.) of $\widehat{m}_{\varphi}(x)$, along with a rate.

Theorem 1. Assume that assumptions (H1), (H3)-(H7) are satisfied.

(i) Under the additional hypothesis (H2), we have

$$
\widehat{m}_{\varphi}(x)-m_{\varphi}(x)=o_{a . c o .}(1) .
$$

(ii) If in addition (H2') is satisfied, we get

$$
\widehat{m}_{\varphi}(x)-m_{\varphi}(x)=O\left(h^{b}\right)+O_{a . c o .}\left(\sqrt{\frac{\ln n}{n \Phi_{x}(h)}}\right) .
$$

Notice that the proof of this theorem is based on a standard decomposition given for all $x \in \mathcal{F}$, by

$$
\widehat{m}_{\varphi}(x)-m_{\varphi}(x)=\frac{1}{m_{0}(x)}\left[\left(m_{1}(x)-E m_{1}(x)\right)-\left(m_{\varphi}(x)-E m_{1}(x)\right)\right]-\frac{m_{\varphi}(x)\left(m_{0}(x)-1\right)}{m_{0}(x)},
$$

where, for $l=0,1$

$$
m_{l}(x)=\frac{1}{n(n-1) E W_{12}(x)} \sum_{i \neq j} W_{i j}(x) \varphi^{l}\left(Y_{j}\right) .
$$

The study of each term of this decomposition can be carried out exactly as done in the proof of Theorem 4.1 and Theorem 4.2 in [1] with replacing $Y$ by $\varphi(Y)$, so for the sake of avoiding repetitions, we omit the proof.

Now, we will focus on the uniform consistency. 


\section{The uniform almost-complete convergence}

\subsection{The estimator $\widehat{m}_{\varphi}$}

We will establish the uniform almost-complete convergence of $\widehat{m}_{\varphi}$ on some subset $S_{\mathcal{F}}$ of $\mathcal{F}$ which can be covered by a finite number of balls. This number has to be related to the radius of these balls (see hypothesis (U5)).

To this goal, let us recall the following definition.

Definition 1. Let $S$ be a subset of a semi-metric space $\mathcal{F}$, and let $\varepsilon>0$ be given. A finite set of points $x_{1}, x_{2}, \ldots, x_{N}$ in $\mathcal{F}$ is called an $\varepsilon$-net for $S$ if $S \subset \bigcup_{k=1}^{N} B\left(x_{k}, \varepsilon\right)$. The quantity $\psi_{S}(\varepsilon)=\ln \left(N_{\varepsilon}(S)\right)$, where $N_{\varepsilon}(S)$ is the minimal number of open balls in $\mathcal{F}$ of radius $\varepsilon$ which is necessary to cover $S$, is called Kolmogorov's $\varepsilon$-entropy of the set $S$.

It is known that the entropy of a set measures its complexity. We refer to [7] and [4] for more details on this topic.

We suppose that $x_{1}, \ldots, x_{N_{r_{n}}\left(S_{\mathcal{F}}\right)}$ is an $r_{n}$-net for $S_{\mathcal{F}}$ where for all $k \in\left\{1, \ldots, N_{r_{n}}\left(S_{\mathcal{F}}\right)\right\}$, $x_{k} \in S_{\mathcal{F}}$ and $\left(r_{n}\right)$ is a sequence of positive real numbers.

In this study, we need the following assumptions.

(U1) There exist a differentiable function $\Phi$ and strictly positive constants $C, C_{1}$ and $C_{2}$ such that

$$
\forall x \in S_{\mathcal{F}}, \forall h>0 ; 0<C_{1} \Phi(h) \leqslant \Phi_{x}(h) \leqslant C_{2} \Phi(h)<\infty
$$

and

$$
\exists \eta_{0}>0, \forall \eta<\eta_{0}, \Phi^{\prime}(\eta)<C,
$$

where $\Phi^{\prime}$ denotes the first derivative of $\Phi$ with $\Phi(0)=0$.

(U2) The generalized regression function $m_{\varphi}$ satisfies:

$$
\exists C>0, \exists b>0, \forall x \in S_{\mathcal{F}}, x^{\prime} \in B(x, h),\left|m_{\varphi}(x)-m_{\varphi}\left(x^{\prime}\right)\right| \leqslant C d^{b}\left(x, x^{\prime}\right) .
$$

(U3) The function $\beta(.,$.$) satisfies (H3) uniformly on x$ and the following Lipschitz's condition

$$
\exists C>0, \forall x_{1} \in S_{\mathcal{F}}, x_{2} \in S_{\mathcal{F}}, x \in \mathcal{F},\left|\beta\left(x, x_{1}\right)-\beta\left(x, x_{2}\right)\right| \leqslant C d\left(x_{1}, x_{2}\right) .
$$

(U4) The kernel $K$ fulfills (H4) and is Lipschitzian on [0,1].

(U5) $\lim _{n \rightarrow \infty} h=0$, and for $r_{n}=O\left(\frac{\ln n}{n}\right)$, the function $\psi_{S_{\mathcal{F}}}$ satisfies for $n$ large enough:

$$
\frac{(\ln n)^{2}}{n \Phi(h)}<\psi_{S_{\mathcal{F}}}\left(\left(\frac{\ln n}{n}\right)<\frac{n \Phi(h)}{\ln n}\right.
$$

and

for some $\beta>1$.

$$
\sum_{n=1}^{\infty} \exp \left\{(1-\beta) \psi_{S_{\mathcal{F}}}\left(\frac{\ln n}{n}\right)\right\}<\infty
$$

(U6) The bandwidth $h$ satisfies: $\exists n_{0} \in \mathbb{N}, \exists C>0$, such that

$$
\forall n>n_{0}, \forall x \in S_{\mathcal{F}}, \frac{1}{\Phi_{x}(h)} \int_{0}^{1} \Phi_{x}(z h, h) \frac{d}{d z}\left(z^{2} K(z)\right)>C>0
$$

and

uniformly on $x$.

$$
h \int_{B(x, h)} \beta(u, x) d P_{X}(u)=o\left(\int_{B(x, h)} \beta^{2}(u, x) d P_{X}(u)\right)
$$


(U7) $\exists C>0$ such that $\forall m \geqslant 2: E\left(|\varphi(Y)|^{m} / X=x\right)<\delta_{m}(x)<C<\infty$ with $\delta_{m}($.$) continuous$ on $S_{\mathcal{F}}$.

Roughly speaking, these hypotheses are uniform version of the assumed conditions in the pointwise case and have already been used in the literature. We refer to [8] for conditions (U1), (U3), (U4) and (U6) and to [4] for assumptions (U2), (U5) and (U7).

The claimed result is as follows.

Theorem 2. Under assumptions (U1)-(U7), we have

$$
\sup _{x \in S_{\mathcal{F}}}\left|\widehat{m}_{\varphi}(x)-m_{\varphi}(x)\right|=O\left(h^{b}\right)+O_{a . c o .}\left(\sqrt{\frac{\psi_{S_{\mathcal{F}}}\left(\frac{\ln n}{n}\right)}{n \Phi(h)}}\right) .
$$

We can readily deduce the uniform consistency of the estimator studied in [1] for which, to the best of our knowledge, only the pointwise convergence is available.

This result shows that, contrary to the finite case, the rate of convergence obtained may differ from that of the pointwise consistency, it is function of the entropy of the subset on which the uniform convergence states.

It is easy to see that the proof of Theorem 2 is a direct consequence of the decomposition (1) and of the following lemmas for which the proofs are relegated to the Appendix.

Lemma 1. Assume that hypotheses (U1), (U2) and (U4) hold, then:

$$
\sup _{x \in S_{\mathcal{F}}}\left|m_{\varphi}(x)-E m_{1}(x)\right|=O\left(h^{b}\right) .
$$

Lemma 2. Under assumptions of Theorem 1, we obtain that:

$$
\sup _{x \in S_{\mathcal{F}}}\left|m_{1}(x)-E m_{1}(x)\right|=O_{a . c o .}\left(\sqrt{\frac{\psi_{S_{\mathcal{F}}}\left(\frac{\ln n}{n}\right)}{n \Phi(h)}}\right) .
$$

Lemma 3. If assumptions (U1),(U3)-(U6) are satisfied, we get:

$$
\sup _{x \in S_{\mathcal{F}}}\left|m_{0}(x)-1\right|=O_{a . c o .}\left(\sqrt{\frac{\psi_{S_{\mathcal{F}}}\left(\frac{\ln n}{n}\right)}{n \Phi(h)}}\right)
$$

and

$$
\sum_{n=1}^{\infty} P\left(\inf _{x \in S_{\mathcal{F}}} m_{0}(x)<\frac{1}{2}\right)<\infty
$$

\subsection{A conditional quantile estimator}

Let $F_{x}(y)=P(Y \leqslant y \mid X=x)$ be the conditional distribution function of $Y$ given $X=x$ where $y$ is real and $x$ is a fixed object in $\mathcal{F}$. To estimate it, we treat this function as a particular case of $m_{\varphi}$ with $\varphi(t)=1_{]-\infty, y]}(t)$ for $y \in \mathbb{R}$. Thus, we estimate $F^{x}(y)$ by

$$
\widehat{F}^{x}(y)=\frac{\sum_{i, j=1}^{n} W_{i j}(x) 1_{\left\{Y_{j} \leqslant y\right\}}}{\sum_{i, j=1}^{n} W_{i j}(x)},
$$

where

$$
W_{i j}(x)=\beta\left(X_{i}, x\right)\left(\beta\left(X_{i}, x\right)-\beta\left(X_{j}, x\right)\right) K\left(h^{-1} d\left(X_{i}, x\right)\right) K\left(h^{-1} d\left(X_{j}, x\right)\right) .
$$


The conditional quantile of order $\alpha(\alpha \in(0,1))$ is $t_{\alpha}(x)=\inf \left\{y \in \mathbb{R}, F^{x}(y) \geqslant \alpha\right\}$. So, we deduce from $\widehat{F}^{x}$ a natural conditional quantile estimator as,

$$
\widehat{t}_{\alpha}(x)=\inf \left\{y \in \mathbb{R}, \widehat{F}^{x}(y) \geqslant \alpha\right\} .
$$

Notice that $t_{1 / 2}(x)$ is the so called conditional median.

To investigate the asymptotic convergence of $\widehat{F}^{x}(y)$, we introduce the following conditions.

(U2)' There exist $\delta>0, C>0$ and $b>0$, such that for any $x \in S_{\mathcal{F}}, x^{\prime} \in B(x, h)$ and $y \in$ $\left[t_{\alpha}(x)-\delta, t_{\alpha}(x)+\delta\right]$, we have

$$
\left|F^{x^{\prime}}(y)-F^{x}(y)\right| \leqslant C d^{b}\left(x, x^{\prime}\right)
$$

(U5)' $\lim _{n \rightarrow \infty} h=0$, and for $r_{n}=O\left(\frac{\ln n}{n}\right)$, the function $\psi_{S_{\mathcal{F}}}$ satisfies for $n$ large enough:

$$
\frac{(\ln n)^{2}}{n \Phi(h)}<\psi_{S_{\mathcal{F}}}\left(\frac{\ln n}{n}\right)<\frac{n \Phi(h)}{\ln n}
$$

and

$$
\sum_{n=1}^{\infty} n^{(\xi+1 / 2)} \exp \left\{(1-\beta) \psi_{S_{\mathcal{F}}}\left(\frac{\ln n}{n}\right)\right\}<\infty
$$

for some $\beta>1$ and $\xi>0$.

The following result concerns the uniform almost complete convergence of $\widehat{F}^{x}(y)$.

Theorem 3. Under assumptions (U1), (U2)', (U3), (U4), (U5)' and (U6), we have

$$
\sup _{x \in S_{\mathcal{F}}} \sup _{y \in\left[t_{\alpha}(x)-\delta, t_{\alpha}(x)+\delta\right]}\left|\widehat{F}^{x}(y)-F^{x}(y)\right|=O\left(h^{b}\right)+O_{a . c o .}\left(\sqrt{\frac{\ln n}{n \Phi(h)}}\right) .
$$

To prove this theorem we make use of the decomposition given, for all $x$ and $y$, by

$$
\widehat{F}^{x}(y)-F^{x}(y)=\frac{1}{m_{0}(x)}\left[\left(\widehat{F}_{N}^{x}(y)-E \widehat{F}_{N}^{x}(y)\right)\left(F^{x}(y)-E \widehat{F}_{N}^{x}(y)\right)\right]-\frac{F^{x}(y)}{m_{0}(x)}\left(m_{0}(x)-1\right)
$$

where $\widehat{F}_{N}^{x}(y)=\frac{1}{n(n-1) E W_{12}(x)} \sum_{i \neq j} W_{i j}(x) 1_{\{Y j \leqslant y\}}$ and $m_{0}(x)$ is defined in (1). Now, it sufficies to apply Lemma 3 together with the following lemmas.

Lemma 4. Assume that hypotheses (U1), (U2)' and (U4) hold, then

$$
\sup _{x \in S_{\mathcal{F}}} \sup _{y \in\left[t_{\alpha}(x)-\delta, t_{\alpha}(x)+\delta\right]}\left|F^{x}(y)-E \widehat{F}_{N}^{x}(y)\right|=O\left(h^{b}\right) .
$$

Lemma 5. Under assumptions of Theorem 3, we obtain that

$$
\sup _{x \in S_{\mathcal{F}}} \sup _{y \in\left[t_{\alpha}(x)-\delta, t_{\alpha}(x)+\delta\right]}\left|\widehat{F}_{N}^{x}(y)-E \widehat{F}_{N}^{x}(y)\right|=O_{a . c o .}\left(\sqrt{\frac{\psi_{S_{\mathcal{F}}}\left(\frac{\ln n}{n}\right)}{n \Phi(h)}}\right) .
$$

The same arguments as in the proof of Lemma 2.8 (resp. Lemma 2.9) in [8] permit us to derive the conclusion of Lemma 4 (resp. Lemma 5).

To obtain the uniform consistency of the conditional quantile estimator, we introduce the following conditions used for example in [8]. 
(U8) $\forall \epsilon>0, \exists \xi>0$ such that for any function $g_{\alpha}$ from $S_{\mathcal{F}}$ into $\left[t_{\alpha}(x)-\delta, t_{\alpha}(x)+\delta\right]$ we have

$$
\sup _{x \in S_{\mathcal{F}}}\left|t_{\alpha}(x)-g_{\alpha}(x)\right| \geqslant \epsilon \text { implies } \sup _{x \in S_{\mathcal{F}}}\left|F^{x}\left(t_{\alpha}(x)\right)-F^{x}\left(g_{\alpha}(x)\right)\right| \geqslant \xi .
$$

(U9) $\exists j>1, \forall x \in S_{\mathcal{F}}, F^{x}$ is $j$-times continuously differentiable on $] t_{\alpha}(x)-\delta, t_{\alpha}(x)+\delta[$ with respect to $y$ and satisfies $F^{x(l)}\left(t_{\alpha}(x)\right)=0$ if $0 \leqslant l<j, F^{x(j)}\left(t_{\alpha}(x)\right)>C>0$ and $F^{x(j)}$ is uniformly continuous on $\left[t_{\alpha}(x)-\delta, t_{\alpha}(x)+\delta\right]$ where $F^{x(l)}$ stands for the $l$ th-order derivative of $F^{x}$.

A known method can be applied to derive the following result from Theorem 3, see for example the proof of Corollary 3.1 in [8].

Corollary 1. Under the hypotheses of Theorem 3 and if (U8)and (U9) are satisfied, we obtain

$$
\sup _{x \in S_{\mathcal{F}}}\left|\widehat{t}_{\alpha}(x)-t_{\alpha}(x)\right|=O\left(h^{b}\right)+O_{\text {a.co. }}\left(\sqrt{\frac{\psi_{S_{\mathcal{F}}}\left(\frac{\ln n}{n}\right)}{n \Phi(h)}}\right) .
$$

\section{A real data application}

In this section, we use a real data set to illustrate the efficacy of the studied method through our conditional median estimator $\widehat{t}_{1 / 2}$. More precisely, we compare this last estimator to two other conditional median estimators: the first is based on the kernel method (denoted KM) and is studied in [6] and the second is based on the local linear method (denoted LLM) and is introduced in [8].

For this purpose, we use the spectrometric data set which can be found at http ://lib.stat. cmu.edu/datasets/tecator. These data consist of 215 pairs $(X i, Y i)_{i=1, \ldots, 215}$. For each $i$, the spectrometric curve $X_{i}$ is the spectra of a finely chopped meat and $Y_{i}$ is the the corresponding fat content obtained by an analytical chemical process. Our goal is to predict the fat content in a piece of meat from its spectrometric curve. For this, we estimate the median $t_{1 / 2}(x)$ of the conditional distribution by $\widehat{t}_{1 / 2}(x)$.

We split these real data into a learning sample containing the first 160 units used to build the estimator and a test sample containing the last 55 units used to predict the fat content and to make a comparison.

The KM (resp. the LLM) estimator is computed with the same parameters as at Subsection 12.4 in [6] (resp. at section 4 in [8]). For the computation of the estimator $\widehat{t}_{1 / 2}(x)$, we use the quadratic kernel $K(x)=\frac{3}{2}\left(1-x^{2}\right) 1_{[0,1]}(x)$, the bandwith $h$ is chosen by a 2 -fold crossvalidation method, the semi-metric $d$ is based on the derivative described in [6] (see routines "semimetric.deriv" in the website http://www.lsp.ups-tlse.fr/staph/npfda) and $\beta=d$.

To illustrate the performance of our estimator, we first plot the true values (provided in the test sample) against the predicted ones by means of the three estimators (one in each graph). This is displayed in Fig. 1. Secondly, to be more precise we evaluate their empirical Mean Square Errors (MSE), defined by

$$
M S E:=\frac{1}{55} \sum_{i=1}^{55}\left(\widehat{Y}_{i}-Y_{i}\right)^{2},
$$

where $Y i$ (resp. $\left.\widehat{Y}_{i}\right)$ is the true (resp. the estimated) value.

The obtained results are

$$
\operatorname{MSE}\left(\widehat{t}_{1 / 2}\right)=3.22, \operatorname{MSE}(\operatorname{LLM})=3.8 \text { and } \operatorname{MSE}(\mathrm{KM})=4.8 \text {. }
$$

This shows that the estimator $\widehat{t}_{1 / 2}$ performs well and that the local linear method seems to improve the quality of the prediction even for functional data. 

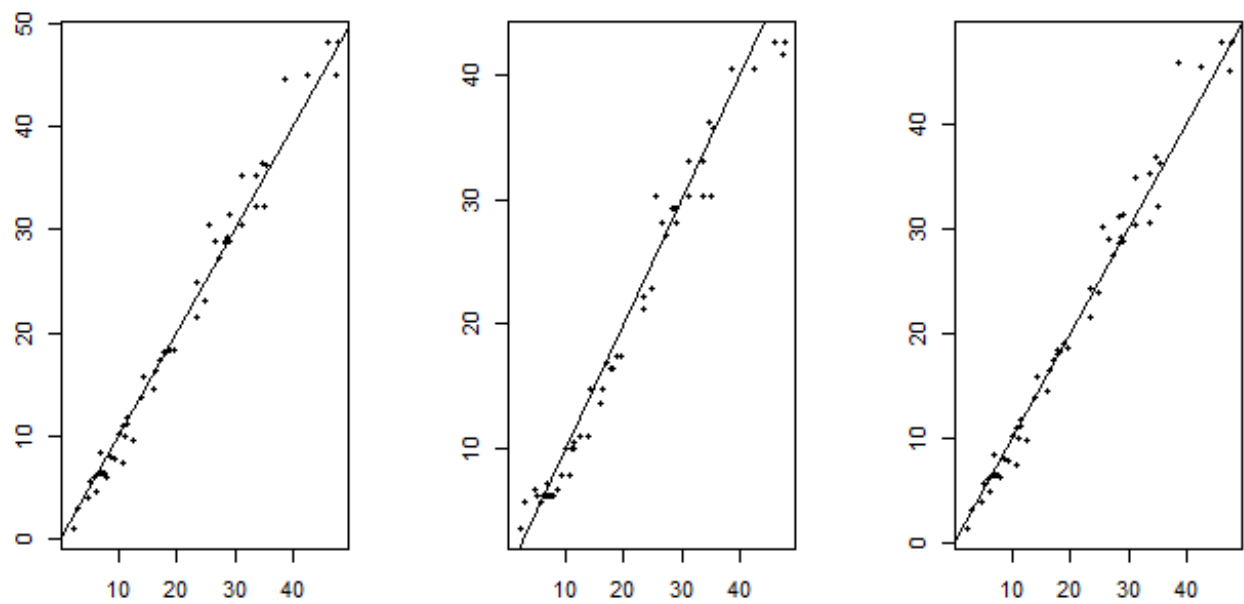

Fig. 1. From left to right: the estimator $\widehat{t}_{1 / 2}$, the KM estimator and the LLM estimator for the spectrometric data

\section{Appendix}

In what follows, let $C$ be some strictly positive generic constant and for any $x \in \mathcal{F}$, and for all $i=1, \ldots, n$ :

$$
K_{i}(x):=K\left(h^{-1} d\left(X_{i}, x\right)\right) \text { and } \beta_{i}(x):=\beta\left(X_{i}, x\right) .
$$

To treat the uniform convergence of $\hat{m}_{\varphi}(x)$, we need to make use of Lemma 4.1 introduced in [8] and stated here as follows.

Lemma 6. Under assumptions (U1),(U3),(U4) and (U6), we obtain that:

i) $\forall(p, l) \in \mathbb{N}^{\star} \times \mathbb{N}, \sup _{x \in S_{\mathcal{F}}} E\left(K_{1}^{p}(x)\left|\beta_{1}^{l}(x)\right|\right) \leqslant C h^{l} \Phi(h)$.

ii) $\inf _{x \in S_{\mathcal{F}}} E\left(K_{1}(x) \beta_{1}^{2}(x)\right)>C h^{2} \Phi(h)$.

Proof of Lemma 1. We have

$$
E m_{l}(x)=\frac{1}{E\left(W_{12}(x)\right)} E\left(W_{12}(x) \varphi^{l}\left(Y_{2}\right)\right),
$$

and $E m_{1}(x)$ can also be written as

$$
E m_{1}(x)=E\left(E\left(m_{1}(x) \mid X_{2}\right)\right)=\frac{1}{E\left(W_{12}(x)\right)} E\left(W_{12}(x) E\left(\varphi\left(Y_{2}\right) \mid X_{2}\right)\right) .
$$

So, we get under assumption (U4)

$$
\left|m_{\varphi}(x)-E m_{1}(x)\right|=\frac{1}{\left|E\left(W_{12}(x)\right)\right|}\left|E\left(W_{12}(x)\left(m_{\varphi}(x)-m_{\varphi}\left(X_{2}\right)\right)\right)\right| \leqslant \sup _{x \prime \in B(x, h)}\left|m_{\varphi}(x)-m_{\varphi}\left(x^{\prime}\right)\right| .
$$

We need to take into account hypothesis (U2) to obtain

$$
\sup _{x \in S_{\mathcal{F}}}\left|m_{\varphi}(x)-E m_{1}(x)\right|=O\left(h^{b}\right) .
$$

Proof of Lemma 2. We use again the following decomposition given in [1]. Namely

$$
m_{1}(x)=Q(x)\left[S_{2,1}(x) S_{4,0}(x)-S_{3,1}(x) S_{3,0}(x)\right],
$$


where, for $p=2,3,4$, and $l=0,1$,

$$
S_{p, l}(x)=\frac{1}{n \Phi_{x}(h)} \sum_{i=1}^{n} \frac{K_{i}(x) \beta_{i}^{p-2}(x) \varphi^{l}\left(Y_{i}\right)}{h^{p-2}}
$$

and

$$
Q(x)=\frac{n^{2} h^{2} \Phi_{x}^{2}(h)}{n(n-1) E\left(W_{12}(x)\right)} .
$$

By following the same steps as in [1], and using lemma 6 instead of lemma A.1 in [1], we obtain under the assumptions (U1)-(U4) and (U6),

$$
Q(x)=O(1), \quad E\left(S_{p, l}(x)\right)=O(1),
$$

uniformly on $\mathrm{x}$, for $p=2,3,4, l=0,1$,

$$
\sup _{x \in S_{\mathcal{F}}}\left|E\left(S_{2,1}(x)\right) E\left(S_{4,0}(x)\right)-E\left(S_{2,1}(x) S_{4,0}(x)\right)\right|=O\left(\frac{1}{n \Phi(h)}\right),
$$

and

$$
\sup _{x \in S_{\mathcal{F}}}\left|E\left(S_{3,1}(x)\right) E\left(S_{3,0}(x)\right)-E\left(S_{3,1}(x) S_{3,0}(x)\right)\right|=O\left(\frac{1}{n \Phi(h)}\right),
$$

which is, in view of hypothesis $(U 5)$, equals to $O\left(\sqrt{\frac{\psi_{S_{\mathcal{F}}}\left(\frac{\ln n}{n}\right)}{n \Phi(h)}}\right)$.

We need to check that for $p=2,3,4$ and $l=0,1$,

$$
\sup _{x \in S_{\mathcal{F}}}\left|S_{p, l}(x)-E(S p, l(x))\right|=O_{a . c o .}\left(\sqrt{\frac{\psi_{S_{\mathcal{F}}}\left(\frac{\ln n}{n}\right)}{n \Phi(h)}}\right) .
$$

To satisfy this aim, let us set

$$
j(x)=\arg \min _{j \in\left\{1,2, \ldots, N_{r_{n}}\left(S_{\mathcal{F}}\right)\right\}} d\left(x, x_{j}\right)
$$

and consider the following decomposition

$$
\begin{aligned}
\sup _{x \in S_{\mathcal{F}}}\left|S_{p, l}(x)-E S_{p, l}(x)\right| & \leqslant \sup _{x \in S_{\mathcal{F}}}\left|S_{p, l}(x)-S_{p, l}\left(x_{j(x)}\right)\right|+ \\
& +\sup _{x \in S_{\mathcal{F}}}\left|S_{p, l}\left(x_{j(x)}\right)-E S_{p, l}\left(x_{j(x)}\right)\right|+ \\
& +\sup _{x \in S_{\mathcal{F}}}\left|E S_{p, l}\left(x_{j(x)}\right)-E S_{p, l}(x)\right|:=F_{1}^{p, l}+F_{2}^{p, l}+F_{3}^{p, l} .
\end{aligned}
$$

Let's, now, study each term $F_{k}^{p, l}$ for $k=1,2,3$.

Study of the terms $F_{1}^{p, l}$ and $F_{3}^{p, l}$.

First, let us analyze the term $F_{1}^{p, l}$. Since $K$ is supported in $[0,1]$ and according to (U1), we 
can write for all $p=2,3,4$

$$
\begin{aligned}
F_{1}^{p, l} \leqslant & \frac{C}{n h^{p-2} \Phi(h)} \sup _{x \in S_{\mathcal{F}}} \sum_{i=1}^{n} \mid K_{i}(x) \beta_{i}^{p-2}(x) \varphi^{l}\left(Y_{i}\right) 1_{B(x, h)}\left(X_{i}\right)- \\
& -K_{i}\left(x_{j(x)}\right) \beta_{i}^{p-2}\left(x_{j(x)}\right) \varphi^{l}\left(Y_{i}\right) 1_{B\left(x_{j(x)}, h\right)}\left(X_{i}\right) \mid \leqslant \\
\leqslant & \frac{C}{n h^{p-2} \Phi(h)} \sup _{x \in S_{\mathcal{F}}} \sum_{i=1}^{n} K_{i}(x) 1_{B(x, h)}\left(X_{i}\right)\left|\varphi^{l}\left(Y_{i}\right)\right|\left|\beta_{i}^{p-2}(x)-\beta_{i}^{p-2}\left(x_{j(x)}\right) 1_{B\left(x_{j(x)}, h\right)}\left(X_{i}\right)\right|+ \\
& +\frac{C}{n h^{p-2} \Phi(h)} \sup _{x \in S_{\mathcal{F}}} \sum_{i=1}^{n} \beta_{i}^{p-2}\left(x_{j(x)}\right) 1_{B\left(x_{j(x)}, h\right)}\left(X_{i}\right)\left|\varphi^{l}\left(Y_{i}\right)\right|\left|K_{i}(x) 1_{B(x, h)}\left(X_{i}\right)-K_{i}\left(x_{j(x)}\right)\right| \\
:= & F_{1.1}^{p, l}+F_{1.2}^{p, l} .
\end{aligned}
$$

Analysis of the term $F_{1.1}^{p, l}$.

According to assumption (U3), we get

$$
\begin{aligned}
& 1_{B(x, h)}\left(X_{i}\right)\left|\beta_{i}(x)-\beta_{i}\left(x_{j(x)}\right) 1_{B\left(x_{j(x)}, h\right)}\left(X_{i}\right)\right| \leqslant \\
& \quad \leqslant C r_{n} 1_{B(x, h) \cap B\left(x_{j(x)}, h\right)}\left(X_{i}\right)+C h 1_{B(x, h) \cap \overline{B\left(x_{j(x)}, h\right)}}\left(X_{i}\right)
\end{aligned}
$$

and

$$
\begin{aligned}
& 1_{B(x, h)}\left(X_{i}\right)\left|\beta_{i}^{2}(x)-\beta_{i}^{2}\left(x_{j(x)}\right) 1_{B\left(x_{j(x)}, h\right)}\left(X_{i}\right)\right| \leqslant \\
& \quad \leqslant C r_{n} h 1_{B\left(x_{j(x)}, h\right)} \cap B(x, h) \\
& \quad\left(X_{i}\right)+C h^{2} 1_{B(x, h) \cap \overline{B\left(x_{j(x)}, h\right)}}\left(X_{i}\right) .
\end{aligned}
$$

By grouping the cases $p=3$ and $p=4$, we found

$$
\begin{aligned}
& 1_{B(x, h)}\left(X_{i}\right)\left|\beta_{i}^{p-2}(x)-\beta_{i}^{p-2}\left(x_{j(x)}\right) 1_{B\left(x_{j(x)}, h\right)}\left(X_{i}\right)\right| \leqslant \\
& \quad \leqslant C r_{n} h^{p-3} 1_{B\left(x_{j(x)}, h\right)} \cap B(x, h)\left(X_{i}\right)+C h^{p-2} 1_{B(x, h) \cap \overline{B\left(x_{j(x)}, h\right)}}\left(X_{i}\right) .
\end{aligned}
$$

which gives the following inequality

$$
\begin{aligned}
F_{1.1}^{P, l} \leqslant \frac{C r_{n}}{n h \Phi(h)} \sup _{x \in \mathcal{S}_{\mathcal{F}}} & \sum_{i=1}^{n}\left|\varphi^{l}\left(Y_{i}\right)\right| K_{i}(x) 1_{B(x, h) \cap B\left(x_{j(x)}, h\right)}\left(X_{i}\right)+ \\
& +\frac{C}{n \Phi(h)} \sup _{x \in \mathcal{S}_{\mathcal{F}}} \sum_{i=1}^{n}\left|\varphi^{l}\left(Y_{i}\right)\right| K_{i}(x) 1_{B(x, h) \cap \overline{B\left(x_{j(x)}, h\right)}}\left(X_{i}\right) .
\end{aligned}
$$

Analysis of the term $F_{1.2}^{p, l}$.

Using the following inequality

$$
\begin{aligned}
& 1_{B\left(x_{j}(x), h\right)}\left(X_{i}\right)\left|K_{i}(x) 1_{B(x, h)}\left(X_{i}\right)-K_{i}\left(x_{j(x)}\right) 1_{B(x, h) \cup \overline{B(x, h)}}\left(X_{i}\right)\right| \leqslant \\
& \quad \leqslant 1_{B(x, h) \cap B\left(x_{j(x)}, h\right)}\left(X_{i}\right)\left|K_{i}(x)-K_{i}\left(x_{j}(x)\right)\right|+K_{i}\left(x_{j}(x)\right) 1_{B\left(x_{j}(x), h\right) \cap \overline{B(x, h)}}\left(X_{i}\right)
\end{aligned}
$$

and by hypotheses (U3) and (U4), we obtain

$$
\begin{aligned}
& \left|\beta_{i}^{p-2}\left(x_{j(x)}\right)\right| 1_{B\left(x_{j(x), h}\right)}\left(X_{i}\right)\left|K_{i}(x) 1_{B(x, h)}\left(X_{i}\right)-K_{i}\left(x_{j(x)}\right)\right| \leqslant \\
& \quad \leqslant C h^{p-2}\left[\frac{r_{n}}{h} 1_{B(x, h) \cap B\left(x_{j(x)}, h\right)}\left(X_{i}\right)+K_{i}\left(x_{j(x)}\right) 1_{B\left(x_{j(x)}, h\right) \cap \overline{B(x, h)}}\left(X_{i}\right)\right],
\end{aligned}
$$


which leads to

$$
\begin{aligned}
F_{1.2}^{p, l} & \leqslant \frac{C r_{n}}{n h \Phi(h)} \sup _{x \in S_{\mathcal{F}}} \sum_{i=1}^{n}\left|\varphi^{l}\left(Y_{i}\right)\right| 1_{B(x, h) \cap B\left(x_{j(x)}, h\right)}\left(X_{i}\right)+ \\
& +\frac{C}{n \Phi(h)} \sup _{x \in S_{\mathcal{F}}} \sum_{i=1}^{n}\left|\varphi^{l}\left(Y_{i}\right)\right| K_{i}\left(x_{j(x)}\right) 1_{\overline{B(x, h)} \cap B\left(x_{j(x)}, h\right)}\left(X_{i}\right) .
\end{aligned}
$$

This last inequality combined with (5) allow us to write

$$
\begin{aligned}
F_{1}^{p, l} & \leqslant \frac{C r_{n}}{n h \Phi(h)} \sup _{x \in S_{\mathcal{F}}} \sum_{i=1}^{n}\left|\varphi^{l}\left(Y_{i}\right)\right| 1_{B(x, h) \cap B\left(x_{j(x)}, h\right)}\left(X_{i}\right)+ \\
& +\frac{C}{n \Phi(h)} \sup _{x \in S_{\mathcal{F}}} \sum_{i=1}^{n}\left|\varphi^{l}\left(Y_{i}\right)\right| K_{i}\left(x_{j(x)}\right) 1_{B\left(x_{j(x)}, h\right) \cap \overline{B(x, h)}}\left(X_{i}\right)+ \\
& +\frac{C}{n \Phi(h)} \sup _{x \in S_{\mathcal{F}}} \sum_{i=1}^{n}\left|\varphi^{l}\left(Y_{i}\right)\right| K_{i}(x) 1_{B(x, h) \cap \overline{B\left(x_{j(x)}, h\right)}}\left(X_{i}\right) .
\end{aligned}
$$

Taking into account hypothesis (U4), we find

$$
F_{1}^{p, l} \leqslant \frac{C r_{n}}{n h \Phi(h)} \sup _{x \in S_{\mathcal{F}}} \sum_{i=1}^{n}\left|\varphi^{l}\left(Y_{i}\right)\right| 1_{B(x, h) \cup B\left(x_{j(x)}, h\right)}\left(X_{i}\right) .
$$

Let

$$
Z_{i}=\frac{C r_{n}\left|\varphi^{l}\left(Y_{i}\right)\right|}{h \Phi(h)} \sup _{x \in S_{\mathcal{F}}} 1_{B(x, h) \cup B\left(x_{j(x)}, h\right)}\left(X_{i}\right) .
$$

The assumption (U7) implies that

$$
E\left|Z_{1}^{m}\right| \leqslant \frac{C r_{n}^{m}}{h^{m} \Phi(h)^{m-1}}
$$

so, by applying corollary A.8 in [6], with $a_{n}^{2}=\frac{r_{n}}{h \Phi(h)}$,

$$
\frac{1}{n} \sum_{i=1}^{n} Z_{i}=E Z_{1}+O_{a . c o .}\left(\sqrt{\frac{r_{n} \ln n}{n h \Phi(h)}}\right) .
$$

Applying (6) again (for $m=1$ ), one gets

$$
F_{1}^{p, l}=O\left(\frac{r_{n}}{h}\right)+O_{a . c o .}\left(\sqrt{\frac{r_{n} \ln n}{n h \Phi(h)}}\right) .
$$

Combining this with assumption (U5) and the second part of the assumption (U1), we obtain

$$
F_{1}^{p, l}=O_{a . c o}\left(\sqrt{\frac{\psi_{S_{\mathcal{F}}}\left(\frac{\ln n}{n}\right)}{n \Phi(h)}}\right) .
$$

Second, since

$$
F_{3}^{p, l} \leqslant E\left(\sup _{x \in S_{\mathcal{F}}}\left|S_{p, l}(x)-S_{p, l}\left(x_{j(x)}\right)\right|\right)
$$

we deduce that

$$
F_{3}^{p, l}=O_{a . c o}\left(\sqrt{\frac{\psi_{S_{\mathcal{F}}}\left(\frac{\ln n}{n}\right)}{n \Phi(h)}}\right) .
$$


Study of the term $F_{2}^{p, l}$.

For all $\eta>0$, we have that

$$
\begin{aligned}
& P\left(F_{2}^{p, l}>\eta \sqrt{\frac{\psi_{S_{\mathcal{F}}}\left(\frac{\ln n}{n}\right)}{n \Phi(h)}}\right)= \\
& =P\left(\max _{j \in\left\{1, \ldots, N_{r_{n}}\left(S_{\mathcal{F}}\right)\right\}}\left|S_{p, l}\left(x_{j(x)}\right)-E\left(S_{p, l}\left(x_{j(x)}\right)\right)\right|>\eta \sqrt{\frac{\psi_{S_{\mathcal{F}}}\left(\frac{\ln n}{n}\right)}{n \Phi(h)}}\right) \leqslant \\
& \leqslant N_{r_{n}}\left(S_{\mathcal{F}}\right) \max _{j \in\left\{1, \ldots, N_{r_{n}}\left(S_{\mathcal{F}}\right)\right\}} P\left(\left|S_{p, l}\left(x_{j(x)}\right)-E\left(S_{p, l}\left(x_{j(x)}\right)\right)\right|>\eta \sqrt{\frac{\psi_{S_{\mathcal{F}}\left(\frac{\ln n}{n}\right)}^{n \Phi(h)}}{n(h)}} .\right.
\end{aligned}
$$

Let us set for $p=2,3,4$ that

$$
\Delta_{p, i}=\frac{1}{h^{p-2} \Phi_{x}(h)}\left[K_{i}\left(x_{j(x)}\right) \beta_{i}^{p-2}\left(x_{j(x)}\right) \varphi^{l}\left(Y_{i}\right)-E\left(K_{i}\left(x_{j(x)}\right) \beta_{i}^{p-2}\left(x_{j(x)}\right) \varphi^{l}\left(Y_{i}\right)\right)\right] .
$$

Using the binomial Theorem, Lemma 6 and hypothesis (U1), (U2) and (U7), gives for $p=2,3,4$,

$$
E\left|\Delta_{p, i}\right|^{m}=O\left(\Phi^{-m+1}(h)\right) .
$$

Therefore, we can apply a Bernstein- type inequality as done in the corollary A-8 in [6], to obtain

$$
P\left(\frac{1}{n}\left|\sum_{i=1}^{n} \Delta_{p, i}\right|>\eta \sqrt{\frac{\psi_{S_{\mathcal{F}}}\left(\frac{\ln n}{n}\right)}{n \Phi(h)}}\right) \leqslant 2 \exp \left(-C \eta^{2} \psi_{S_{\mathcal{F}}}\left(\frac{\ln n}{n}\right)\right) .
$$

Thus, by choosing $\beta$ such that $C \eta^{2}=\beta$, we get

$$
P\left(F_{2}^{p, l}>\eta \sqrt{\frac{\psi_{S_{\mathcal{F}}}\left(\frac{\ln n}{n}\right)}{n \Phi(h)}}\right) \leqslant C N_{r_{n}}\left(S_{\mathcal{F}}\right)^{1-\beta}
$$

Then, hypothesis (U5) allows us to write

$$
F_{2}^{p, l}=O_{a . c o .}\left(\sqrt{\frac{\psi_{S_{\mathcal{F}}}\left(\frac{\ln n}{n}\right)}{n \Phi(h)}}\right) .
$$

Finally, the result of Lemma 2 follows from the relations (7), (9) and (8).

Proof of Lemma 3. The first part of the claimed results can be directly deduced from the proof of Lemma 2 by taking, for all $i, \varphi\left(Y_{i}\right)=1$. For the second part, It comes straightforward that

$$
\begin{aligned}
\inf _{x \in S_{\mathcal{F}}} m_{0}(x)<\frac{1}{2} & \Rightarrow \exists x \in S_{\mathcal{F}} \text { such that } 1-m_{0}(x)>\frac{1}{2} \Rightarrow \sup _{x \in S_{\mathcal{F}}}\left|1-m_{0}(x)\right|>\frac{1}{2} \\
& \Rightarrow \sum_{n=0}^{\infty} P\left(\inf _{x \in S_{\mathcal{F}}} m_{0}(x)<\frac{1}{2}\right)<\infty .
\end{aligned}
$$

\section{References}

[1] J.Barrientos-Marin, F.Ferraty, P.Vieu, Locally modelled regression and functional data, J.ournal of Nonparametric Statistics, 22(2010), 617-632. 
[2] K.Benhenni, F.Ferraty, M.Rachdi, P.Vieu, Local smoothing regression with functional data, Computational Statistics, 22(2007), no. 3, 353-369.

[3] J.Demongeot, A.Laksaci, F.Madani, M.Rachdi, Functional data analysis: conditional density estimation and its application, Statistics, 47(2013), no. 1, 26-44.

[4] F.Ferraty, A.Laksaci, A.Tadj, P.Vieu, Rate of uniform consistency for nonparametric estimates with functional variables. Journal of Statistical planning and inference, 140(2010), $335-352$.

[5] F.Ferraty, I.Van Keilegom, P.Vieu, On the validity of the bootstrap in nonparametric functional regression, Scandinavian Journal of Statistics, 37(2008), no. 2, 286-306.

[6] F.Ferraty, P.Vieu, Nonparametric functional data analysis. Theory and Practice. Springer Series in Statistics, New York, 2006.

[7] A.N.Kolmogorov, V.M.Tikhomirov, $\varepsilon$-entropy and "-capacity", Uspekhi Matematicheskikh Nauk, 14(1959), 3-86 (in Russian).

[8] F.Messaci, N.Nemouchi, I.Ouassou, M.Rachdi, Local polynomial modelling of the conditional quantile for functional data, Statistics Methods and Applications, 24(2015), no. 4, $597-622$.

\title{
Класс локальных линейных оценок с функциональными данными
}

\author{
Сара Леулми \\ Фатиха Мессачи \\ Факультет математики \\ Университет братьев Ментури \\ дорога Айн-эль-Бея, Константин, 25017 \\ Алжир
}

\begin{abstract}
$\overline{\text { Введем локальную линейную непараметрическую оценку для обобщенной функции регрессии ска- }}$ лярной переменной отклика для заданной случайной величины, принимающей значения в полуметрическом пространстве. Мы устанавливаем скорость равномерной согласованности для предлагаемых оченок. Затем, основываясь на реальном наборе данных, мы проиллюстрируем эфбективность конкретного изученного оченщика по сравнению с другими известными оценщиками.
\end{abstract}

Ключевые слова: локально моделируемая регрессия, непараметрическая оченка, скорость сходимости, равномерная почти полная сходимость. 